\title{
Erratum to: Influence of particle size on the melting characteristics of organic compounds
}

\author{
Frédéric Rosa $^{1,2} \cdot$ Yohann Corvis $^{1,2} \cdot$ René Lai-Kuen $^{3} \cdot$ Christine Charrueau $^{4}$. \\ Philippe Espeau ${ }^{1,2}$
}

Published online: 6 May 2015

(C) Akadémiai Kiadó, Budapest, Hungary 2015

\section{Erratum to: J Therm Anal Calorim (2015) \\ 120:783-787 \\ DOI 10.1007/s10973-014-4210-8}

The correct present address for Frédéric Rosa, Yohann Corvis and Philippe Espeau is provided here.

The online version of the original article can be found under doi:10.1007/s10973-014-4210-8.

Philippe Espeau

philippe.espeau@parisdescartes.fr

1 EA 4066 «Physico-Chimie Industrielle du Médicament», Faculté des Sciences Pharmaceutiques et Biologiques,

Université Paris Descartes, 4 Avenue de l'Observatoire, 75006 Paris, France

2 Present Address: Unité de Technologies Chimiques et Biologiques pour la Santé, U 1022 INSERM, UMR 8258

CNRS, Faculty of Pharmacy, University Paris Descartes, Sorbonne Paris Cité, 4 avenue de l'Observatoire, 75006 Paris, France

3 Unité de Services US25 (INSERM), Plateau Technique Imagerie Cellulaire et Moléculaire (PT-ICM), Faculté des Sciences Pharmaceutiques et Biologiques, Université Paris Descartes, Sorbonne Paris Cité, 4 avenue de l'Observatoire, 75006 Paris, France

4 Unité de Technologies Chimiques et Biologiques pour la Santé, Inserm U 1022 CNRS UMR 8258, Faculté des Sciences Pharmaceutiques et Biologiques, Université Paris Descartes, Sorbonne Paris Cité, 4 avenue de l'Observatoire, 75006 Paris, France 